\title{
Detection of Hepatitis B Virus-Host Junction Sequences in Urine of Infected Patients
}

\author{
Selena Y. Lin, ${ }^{1}$ Yih-Ping Su, ${ }^{2}$ Evan R. Trauger, ${ }^{1}$ Benjamin P. Song, ${ }^{1}$ Emilie G.C. Thompson, ${ }^{2}$ Malcolm C. Hoffman, ${ }^{1}$ \\ Ting-Tsung Chang, ${ }^{3}$ Yih-Jyh Lin, ${ }^{4}$ Yu-Lan Kao, ${ }^{2}$ Yixiao Cui, ${ }^{2}$ Hie-Won Hann, ${ }^{5}$ Grace Park,,${ }^{5}$ Fwu-Shan Shieh, ${ }^{1}$ Wei Song, ${ }^{1}$ and \\ Ying-Hsiu $\mathrm{Su}^{2}$
}

\begin{abstract}
Integrated hepatitis B virus (HBV) DNA, found in more than $85 \%$ of $\mathrm{HBV}$-associated hepatocellular carcinomas (HBV-HCCs), can play a significant role in HBV-related liver disease progression. HBV-host junction sequences (HBV$\mathrm{JSs}$ ), created through integration events, have been used to determine HBV-HCC clonality. Here, we investigate the feasibility of analyzing HBV integration in a noninvasive urine liquid biopsy. Using an $\mathrm{HBV}$-targeted next-generation sequencing (NGS) assay, we first identified HBV-JSs in eight HBV-HCC tissues and designed short-amplicon junction-specific polymerase chain reaction assays to detect HBV-JSs in matched urine. We detected and validated tissue-derived junctions in five of eight matched urine samples. Next, we screened 32 urine samples collected from 25 patients infected with HBV (5 with hepatitis, 10 with cirrhosis, 4 with HCC, and 6 post-HCC). Encouragingly, all 32 urine samples contained HBV-JSs detectable by HBV-targeted NGS. Of the 712 total HBV-JSs detected in urine, 351 were in gene-coding regions, 11 of which, including TERT (telomerase reverse transcriptase), had previously been reported as recurrent integration sites in HCC tissue and were found only in the urine patients with cirrhosis or HCC. The integration breakpoints of HBV DNA detected in urine were found predominantly $(\sim 70 \%)$ at a previously identified integration hotspot, HBV DR1-2 (down-regulator of transcription 1-2). Conclusion: HBV viral-host junction DNA can be detected in urine of patients infected with HBV. This study demonstrates the potential for a noninvasive urine liquid biopsy of integrated HBV DNA to monitor patients infected with HBV for HBV-associated liver diseases and the efficacy of antiviral therapy. (Hepatology Communications 2021;5:1649-1659).
\end{abstract}

$\mathrm{H}$ epatitis B virus (HBV) integration can occur early in the course of infection. ${ }^{(1-3)}$ Integration events are known to cause insertional mutagenesis, chromosomal instability, activation of oncogenes, and inflammation, and thus can play a critical role in liver disease progression and the development of hepatocellular carcinoma (HCC). ${ }^{(4)}$ Integrated viral DNA has been detected in more than $85 \%$ of $\mathrm{HBV}-$ associated HCC (HBV-HCC) cases. $^{(5-8)}$ Although $\mathrm{HBV}$ integration sites have been observed throughout the human genome, integration into known HCC driver genes (such as TERT [telomerase reverse

Abbreviations: bp, base pair; $c f D N A$, cell-free DNA; DR1-2, down-regulator of transcription 1-2; dsDNA, double-stranded DNA; FOCAD, focadhesin; $H B V$, hepatitis B virus; $H B V$-HCC, HBV-infected hepatocellular carcinoma; $H B V$-JS, HBV-host junction sequence; HCC, hepatocellular carcinoma; NGS, next-generation sequencing; PCR, polymerase chain reaction; PPP2R2C, protein phosphatase 2 regulatory subunit Bgamma; $R E$, restriction endonuclease; $S R$, supporting read; TERT, telomerase reverse transcriptase; UMT, unique molecular tag.

Received February 9, 2021; accepted June 20, 2021.

Additional Supporting Information may be found at onlinelibrary. wiley.com/doi/10.1002/hep4.1783/suppinfo.

Supported by the National Cancer Institute (R43-CA165312, R43-CA192507, and R44-CA165312).

(C) 2021 The Authors. Hepatology Communications published by Wiley Periodicals LLC on behalf of American Association for the Study of Liver Diseases. This is an open access article under the terms of the Creative Commons Attribution-NonCommercial-NoDerivs License, which permits use and distribution in any medium, provided the original work is properly cited, the use is non-commercial and no modifications or adaptations are made.

View this article online at wileyonlinelibrary.com.

DOI 10.1002/hep4.1783

Potential conflict of interest: Dr. Selena Lin owns stock in, is employed by, and received grants from JBS Science. Dr. Wei Song owns stock in and is the CEO of JBS Science. Dr. Ying-Hsiu Su owns stock and equity in and advises JBS Science. Her spouse is CEO of JBS Science. 
transcriptase], MLL4 [mixed-lineage leukemia-like protein], and CCNE1 [cyclin E1]) has been reported repeatedly in patients with $\mathrm{HCC}{ }^{(9)}$

One outcome of HBV-DNA integration is the creation of a unique $\mathrm{HBV}$-host junction sequence (HBV-JS), representing a specific molecular signature of the infected cell. HBV-DNA integrations detected in tissue have been used to determine the clonality of recurrent HCC tumors. ${ }^{(10,11)}$ Recent studies have shown that integrated HBV DNA can be detected in the circulation as well. ${ }^{(12-15)} \mathrm{We}$ have previously demonstrated that urine of patients with HCC contains cell-free DNA (cfDNA) with HCC-associated modifications, ${ }^{(16-19)}$ whereas urine of patients infected with $\mathrm{HBV}$ contains fragmented (but not intact) HBV DNA. ${ }^{(20)}$ We hypothesize that urine can serve as a noninvasive liquid biopsy for the investigation of $\mathrm{HBV}$ integration in patients infected with HBV.

In this proof-of-concept study, we assessed the feasibility of detecting HBV-JSs in urine from patients infected with $\mathrm{HBV}$. We first identified HBV-JSs in HCC tissue, followed by detection in matched urine by sensitive junction-specific polymerase chain reaction (PCR) assays. Next, we detected $\mathrm{HBV}-\mathrm{JS}$ s in urine from patients infected with $\mathrm{HBV}$ with hepatitis, cirrhosis, and $\mathrm{HCC}$, including those undergoing recurrence monitoring (post-HCC), using an HBV-targeted nextgeneration sequencing (NGS) assay. These findings support the potential of urine as a noninvasive liquid biopsy for integrated HBV DNA, which can be used to monitor the dynamics of $\mathrm{HBV}$ integration in the infected liver and to assess HBV treatment efficacy and disease progression.

\section{Patients and Methods}

\section{STUDY SUBJECTS AND SPECIMENS}

Two studies, A and B, were performed. Study A specimens included archived DNA isolated from eight pairs of matched HBV-HCC formalin-fixed paraffinembedded tumor tissue and urine samples. The specimens were obtained from patients in stages I-II undergoing surgery (Table 1) at the National ChengKung University Hospital, Taiwan, as described previously. ${ }^{(17,19)}$ Study B was performed on urine samples collected from 25 patients infected with $\mathrm{HBV}$, including 5 with hepatitis, 10 with cirrhosis, 4 with HCC, and 6 post-HCC, at the Thomas Jefferson University Hospital (Table 2). Five patients (B7, B15, B17, B19, and B20) provided more than one urine collection over the course of the study, resulting in a total of 32 urine samples. Additionally, urine samples from $8 \mathrm{HBV}$-negative donors (4 male, 4 female), aged 23-60 years, were collected and served as controls. All samples were collected with written, informed consent and in accordance with the guidelines of the institutional review board.

\section{URINE COLLECTION AND DNA ISOLATION}

Urine samples were collected and total urine DNA isolated, as previously described. ${ }^{(21)}$ For study A, cfDNA was obtained by isolating the $<1-\mathrm{kb}$ fraction from total urine DNA using carboxylated magnetic beads, a method previously developed by our laboratory. ${ }^{(22)}$ For study B, the JBS urine cfDNA isolation kit (JBS Science Inc., Doylestown, PA) was used according to the manufacturer's specifications.

\section{ARTICLE INFORMATION:}

From the ${ }^{1}$ JBS Science, Inc., Doylestown, PA, USA; ${ }^{2}$ The Baruch S. Blumberg Research Institute, Doylestown, PA, USA; ${ }^{3}$ Department of Internal Medicine, National Cheng Kung University Hospital, College of Medicine, Tainan, Taiwan, Republic of China; ${ }^{4}$ Department of Surgery, National Cheng Kung University Hospital, College of Medicine, Tainan, Taiwan, Republic of China; ${ }^{5}$ Liver Disease Prevention Center, Division of Gastroenterology and Hepatology, Thomas Jefferson University Hospital, Philadelphia, PA, USA.

\section{ADDRESS CORRESPONDENCE AND REPRINT REQUESTSTO:}

Ying-Hsiu Su, Ph.D.

The Baruch S. Blumberg Research Institute

3805 Old Easton Road
Doylestown, PA 18902, USA

E-mail: Ying-hsiu.su@bblumberg.org

Tel.: +1-215-489-4949 


\section{TABLE 1. CHARACTERISTICS OF ARCHIVED DNA FROM MATCHED HBV-HCC TISSUE AND URINE FOR} STUDY A

\begin{tabular}{lccccc} 
Patient ID & Age (Years) & Sex (M/F) & Cirrhosis & Tumor stage* & Tumor Size (cm) \\
\hline A1 & 57 & M & - & 1 & 4.0 \\
A2 & 29 & M & + & 2 & 7.0 \\
A3 & 42 & M & + & 1 & 2.0 \\
A4 & 49 & M & + & 2 & 3.4 \\
A5 & 61 & M & - & 2 & 2.3 \\
A6 & 75 & F & - & 1 & 3.0 \\
A7 & 47 & M & - & 1 & 4.5 \\
A8 & 39 & F & - & 2 & 10.0
\end{tabular}

*HCC tumors were staged using the tumor-node-metastasis staging system. Abbreviations: F, female; $\mathrm{M}$, male.

\section{HBV-JS DETECTION BY HBV-TARGETED NGS ASSAY AND VALIDATION BY PCR-SANGER SEQUENCING}

Tissue DNA was fragmented by sonication and subjected to Illumina (San Diego, CA) NGS library preparation, as previously described. ${ }^{(23)}$ Ten cycles of library amplification with Herculase II Fusion polymerase were performed (Agilent Technologies, Santa Clara, CA). Urine cfDNA samples underwent NGS library preparation using the ThruPLEX Tag-Seq kit (Cat\# R400585; Takara Bio, Mountain View, CA), which contains unique molecular tags (UMTs) and uses double-stranded DNA (dsDNA) as a substrate, according to manufacturer specifications. Of note, the TruPLEX Tag-Seq kit converted dsDNA at least 300 times more efficiently than single-stranded DNA (ssDNA) when control templates of 131-nucleotide (nt) ssDNA and 140-base pair (bp) dsDNA were used to validate the performance of the kit. Library DNA was subjected to an HBV-targeted NGS assay following the manufacturer's instructions (JBS Science). The captured library DNA was pooled for NGS analysis on the Illumina MiSeq platform (Penn State Hershey Genomics Sciences Facility at Penn State College of Medicine, Hershey, PA) or on the NovaSeq platform (Psomagen Inc., Rockville, MD).

The ChimericSeq analysis software we developed previously ${ }^{(24)}$ was used to detect $\mathrm{HBV}-\mathrm{JS}$ s in NGS data sets. For NGS libraries containing UMTs, UMT family consolidation and consensus read generation were performed using the software package Connor. ${ }^{(25)}$ Briefly, UMT-containing reads were aligned to human and HBV reference genomes, and paired reads were grouped into families based on the mapping coordinates and combined UMT sequence. For each UMT family, a consensus read pair was generated. The consensus reads were then processed by ChimericSeq for HBV-JS identification.

To validate junctions identified by NGS, junctionspecific primers were designed for the host and viral sequences for tissue and urine (Supporting Table S1). Primers used for generating PCR products larger than $100 \mathrm{bp}$ for Sanger sequencing were only used for tissue junction validation, whereas urine primers were designed to target the same junction sequence generating amplicons of 65 bp or less. The junctionspecific PCR was performed with Hotstart Plus Taq Polymerase (Qiagen, Valencia, CA). PCR products were visualized by agarose gel electrophoresis on a 2.2\% FlashGel DNA Cassette (Lonza Group, Basel, Switzerland) and subsequently subjected to Sanger sequencing, a nested PCR reaction, or restriction endonuclease (RE) digestion using enzymes obtained from New England Biolabs (Ipswich, MA), according to the manufacturer's specifications.

To control for artifacts generated by the HBVtargeted NGS assay, which may be unavoidable with short PCR templates, an artifact library was generated using as input a mix of $30 \mathrm{ng}$ of sonicated (predominantly 170-200-bp fragments) normal human genomic DNA and 9,000 copies of HBV plasmid DNA. The mix contained 10-100 times more copies of HBV DNA than the $>500$ urine cfDNA samples examined (including previously described samples ${ }^{(20)}$ ), in which HBV-DNA content ranged from undetectable to hundreds of copies per milliliter of urine (as 


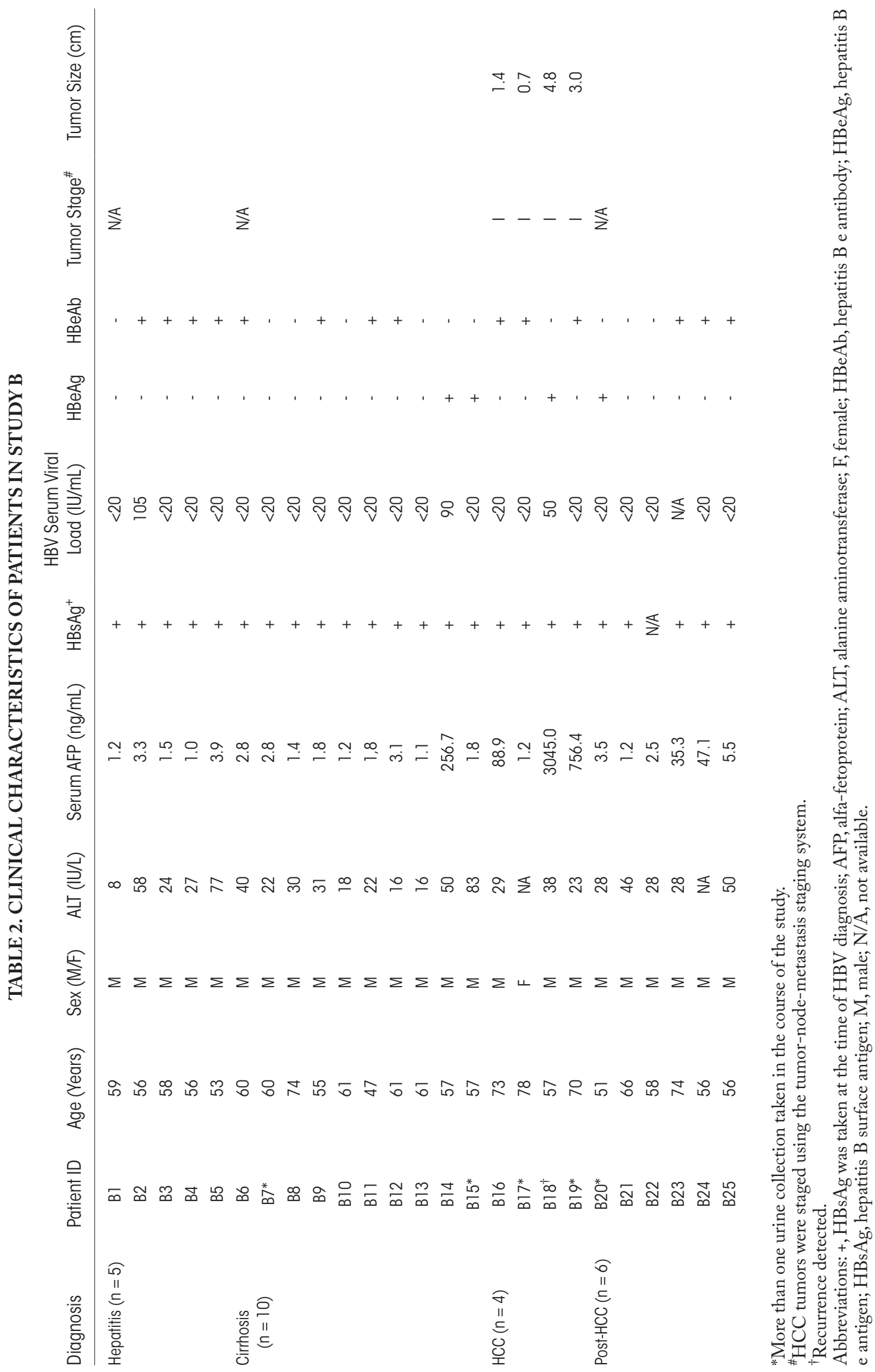


determined by quantitative PCR). The artifact library was used to remove HBV-JSs from each test sample (Supporting Table S2).

\section{DATA AVAILABILITY STATEMENT}

The sequence data for the subjects studied in this work who consented to data archiving have been deposited in the NCBI Sequencing Read Archive (accession no. PRJNA720423).

\section{STATISTICAL ANALYSIS}

The chi-square test was performed for categorical variables. For all statistical tests, the level of significance was defined as $P<0.05$ to determine whether differences were significant.

\section{Results}

\section{DETECTION OF HBV \\ INTEGRATION IN URINE DNA BY JUNCTION-SPECIFIC PCR ASSAYS}

To investigate whether HBV-JSs can be detected in urine, we identified eight pairs of archived DNA samples from matched tissue and urine from patients with HBV-HCC (study A; Table 1), with each urine DNA isolate extracted from at least $1 \mathrm{~mL}$ of urine. We first detected HBV-JSs in tissue DNA using an HBVtargeted NGS assay, as described previously. HBV integrations were detected in all eight HCC tumor DNA samples (Table 3). The affected regions included the TERT gene, the most frequently reported integrantcontaining host gene (patient A5). ${ }^{(9)}$ The junctions were validated by Sanger sequencing (Supporting Fig. S1) using primers listed in Supporting Table S1.

To detect HCC tissue-derived $\mathrm{HBV}$ junction DNA in urine, we designed a junction-specific PCR assay for each junction (Supporting Table S1). The amplicons were designed to be short, as urine cfDNA is mostly fragmented to less than $250 \mathrm{bp} .{ }^{(21)}$ Matched tissue and urine DNA samples were subjected to junction-specific PCR amplification. PCR products of expected size were generated from five of the eight matched urine samples tested. To determine whether the PCR products generated from the urine and tissue in each of the five tissue-urine pairs were identical, we used either two-step nested PCR followed by RE digestion of $\mathrm{PCR}$ products (patients A5, A6, and A8; Fig. 1A) or Sanger sequencing when no suitable RE site was available in PCR products (patients $\mathrm{A} 1$ and A2; Fig. 1B-D). Four (patients A2, A5, A6, and A8) of the five PCR product pairs contained sequences indistinguishable by these methods, indicating that the HCC tissue-derived HBV-JS was present in the matched urine.

Interestingly, the sizes of the junction PCR products from patient $\mathrm{A} 1$ were different between tissue and urine (Fig. 1C). Sanger sequencing analysis identified a 23-nt insert, indicating a re-arrangement between chromosomes 5 and 10 in the urine DNA sample. To determine whether the urine-identified integration site could be found in tumor-tissue DNA, we

TABLE 3. HBV INTEGRATIONS IDENTIFIED IN ARCHIVED TUMOR-TISSUE DNA OF STUDY A PATIENTS

HBV-Host Junction Breakpoint Nucleotide Position*

\begin{tabular}{|c|c|c|c|c|}
\hline \multirow[b]{2}{*}{ Patient ID } & \multirow[b]{2}{*}{ HBV-Host Junction Sequences } & & \multirow[b]{2}{*}{ Gene } \\
\hline & & HBV & Human & \\
\hline $\mathrm{Al}$ & ccttgaggcgtacttcaaaaCCCAGACCCAGCT & 1712 & Chr.10: 31192695 & LOC101929352 \\
\hline A2 & aggagtgttatggtgtctcaTAAATGGTGATATAA & 247 & Chr.1: 165248017 & None \\
\hline A3 & gttgggggagggactagCTCATTAATCATTGT & 1755 & Chr.11:92048629 & LINE2 \\
\hline A4 & gcaccatgcaactttttTCCGAACCTGTGTACT & 1825 & Chr. 16: 30207023 & None \\
\hline A5 & ttgtactaggaggctgCATGGCCGGAAGTCT & 1783 & Chr.5: 1292170 & TERT \\
\hline A6 & ctgtagg cataaattgCCTACAGCAATGTATA & 1796 & Chr.14: 67004392 & GPHN \\
\hline A7 & gatctttgtactaggaggAACATGCCCAAGAA & 1780 & Chr.5: 165559760 & None \\
\hline A8 & agactgtgtgtttaaCTCATCTGTCCAAACCC & 1725 & Chr. 14: 103176895 & None \\
\hline
\end{tabular}

Note: Lowercase sequences represent HBV DNA. Underlined and capitalized sequences represent human DNA. Underlined, lowercase, bold bases represent overlapping human/HBV sequences. "None" indicates that no known gene was present within $100 \mathrm{~kb}$ of the integration site.

*HBV accession \#NC_003977.2; human genome GRCh38. 


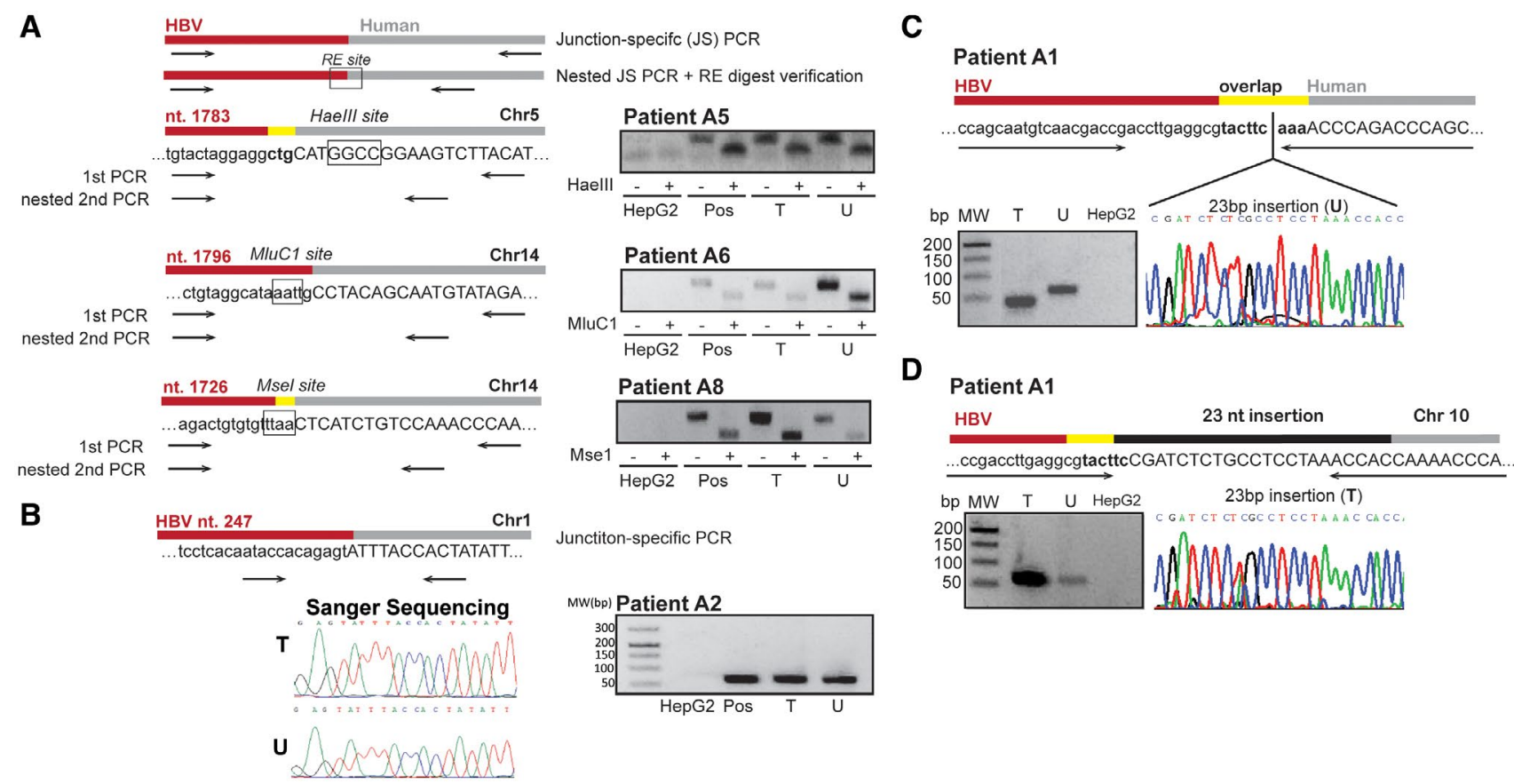

FIG. 1. Detection of HBV-host junction sequences in study A, matched archived tissue, and urine DNA. (A) RE digestion approach. Tissue and urine DNA was amplified using two-step nested junction-specific PCR. The products generated by HBV and human primers were incubated in the absence (-) or presence (+) of the respective RE and analyzed by gel electrophoresis. The expected RE-digested PCR product sizes are patient A5 (25 bp and $20 \mathrm{bp}$ of the 45-bp PCR product), A6 (24 bp and 26 bp of the 53-bp PCR product), and A8 (22 bp and $36 \mathrm{bp}$ of the $57-\mathrm{bp}$ PCR product, but the 22-bp band was not included in the picture). (B) Sanger sequencing approach. The junction sequence of patient A2 was generated by PCR amplification of tissue and urine DNA using HBV and human primers. (C) Sequence of the HBV-host junction at chromosome 10 (Chr10) in the urine of patient A1. Sanger sequencing of the urine-derived junction PCR product revealed a 23-nt insertion compared with the tissue product, as shown by gel electrophoresis. (D) Detection of the 23-nt inserted $\mathrm{HBV}$-host junction in the corresponding tissue. Using a hybrid Chr5-Chr10 primer, a specific tissue junction PCR product was obtained and found to be identical by sequencing to the urine-derived 23-nt insertion. Tissue DNA and HepG2 DNA served as positive and negative controls, respectively. Abbreviations: MW, molecular weight; Pos, positive; T, tissue; U, urine.

designed a primer across the chimeric chromosome $5 / 10$ sequence (Fig. 1D) to specifically amplify the $23-$ nt insert-containing HBV-JS species. Remarkably, the tripartite HBV-JS was successfully amplified and validated in the matched tissue DNA sample (Fig. 1D). In total, we were able to detect and validate five of the eight HCC-tissue-derived HBV-JSs in matched urine samples.

\section{DETECTION AND \\ CHARACTERIZATION OF HBV-JSs IN THE URINE OF HBV-INFECTED PATIENTS BY AN HBV-TARGETED NGS ASSAY}

After detecting liver-derived HBV-JSs in the urine of patients with $\mathrm{HBV}-\mathrm{HCC}$ by junction-specific
PCR assays, we next assessed whether it was feasible to analyze $\mathrm{HBV}$ integrations in urine without prior knowledge of junction sequences from tumor tissue and before disease progression to HCC. A total of 32 urine specimens were collected from 25 $\mathrm{HBV}$-infected individuals, including 5 patients with hepatitis, 10 with cirrhosis, 4 with HCC, and 6 postHCC (study B; summarized in Table 2). Five patients provided more than one urine specimen in the course of the study. For HBV-negative controls, we included urine samples from 4 males and 4 females who had no known history of $\mathrm{HBV}$ infection. Urine DNA was isolated, enriched for HBV sequences, and subjected to NGS followed by HBV-JS identification as described previously. We used a threshold of two unique (UMT-consolidated) supporting reads (SRs) for junction detection for two reasons. First, this cutoff 
is consistent with other studies of $\mathrm{HBV}$ integration in HCC tissue. ${ }^{(8,26-28)}$ Second, the PCR-Sanger sequencing validation rate we obtained in a previous study of $16 \mathrm{HBV}-\mathrm{HCC}$ tumor tissue samples was $88 \%$, which is in line with validation rates reported in the literature. ${ }^{(23,26,28)}$ No HBV-JSs with two or more SRs were observed in the NGS data obtained from the $8 \mathrm{HBV}$-negative controls. In contrast, 31 of 32 $\mathrm{HBV}$-infected urine samples contained $\mathrm{HBV}$-JSs at this cutoff.

Of the total of 712 HBV-JSs identified, 84, 369, 41 , and 218 junctions were detected in 4 hepatitis, 12 cirrhosis, $6 \mathrm{HCC}$, and 9 post-HCC urine samples, respectively. Table 4 lists the major HBV-JSs of each individual urine sample, defined as the two most abundant HBV-JSs supported by at least 3\% of total junction reads. As expected, we detected integrations in both gene-coding and noncoding regions in all disease categories. The latter included repeat regions, such as LINEs, SINEs, and simple repeats, ${ }^{(29)}$ which have been previously reported as $\mathrm{HBV}$ integration sites.

Among the targets of the 351 unique integrations in gene-coding regions detected in urine, 11 genes had been previously reported in liver tissue from patients with severe liver diseases, such as cirrhosis and HCC. These genes are AC007880.1, ADAM12 [disintegrin and metalloproteinase domain-containing protein 12], ARSA [arylsulfatase A], ATF6 [activating transcription factor 6], CLEC2L [C-type lectin domain family 2 member L], FOCAD [focadhesin], PPP2R2C [protein phosphatase 2 regulatory subunit Bgamma], TNFRSF9 [TNF receptor superfamily member 9], TXNDC16 [thioredoxin domain containing 16], $V E G F C$ [vascular endothelial growth factor C], and TERT. Most interestingly, all of the genes, except for $A C 007880.1$ and $A R S A$, have been associated with the development of HCC (ADAM12, ${ }^{(30)} A T F 6,{ }^{(31)}$ $C L E C 2 L,{ }^{(32)} T E R T$, and $\left.V E G F C^{(33)}\right)$ or other cancers (FOCAD, ${ }^{(34)}$ PPP2R2C, ${ }^{(35)}$ TNFRSF9, ${ }^{(36)}$ and TXNDC16 $\left.{ }^{(37)}\right)$.

\section{DISTRIBUTION OF INTEGRATED HBV BREAKPOINTS DETECTED IN URINE}

We next examined the HBV genome breakpoints of the 712 junction sequences detected in urine. The $\mathrm{HBV}$ sequences of these junctions were plotted based on their locations in the HBV genome (Fig. 2A), and the SR statistics and HBV breakpoint regions are summarized in Fig. 2B. The number of SRs for a given junction varied widely within each disease type. Of the 712 junctions detected, $70 \%$ had two SRs. Approximately 70\% of HBV-DNA breakpoints detected in urine were clustered in the HBV DR1-2 region, a known integration hotspot ${ }^{(8)}$ (Fig. 2B). The second-most frequent of the previously reported $\mathrm{HBV}$ breakpoint regions, PreS, ${ }^{(8)}$ was also identified in urine, with higher frequencies found in hepatitis (7.1\%), cirrhosis (10.5\%), and post-HCC (7.3\%) as compared with HCC (4.9\%). However, the association of integrated HBV breakpoint location (DR1-2, PreS1/2, or other) with disease type was not statistically significant $\left(\chi^{2}[6,712=6.3] ; P=0.39\right)($ Fig. $2 \mathrm{~B})$.

\section{PROFILING OF DETECTABLE HBV INTEGRATION SITES IN SERIALLY COLLECTED URINE SAMPLES}

Urine cfDNA containing HBS-JSs may reflect the $\mathrm{HBV}$ integration profile of the infected liver. For a preliminary assessment of the possibility of using HBS-JSs for disease monitoring, we collected serial urine samples from 5 patients (B7, B15, B17, B19, and B20) and compared the HBV-JSs detected in different collections (Table 4). Patients B15 and B19, both with cirrhosis, had two collections 1 week apart and showed consistent integration in SINE and simple repeat regions, respectively, although the integration breakpoints were different. This finding indicates that the detection of these junctions does not reflect clonal expansion, but rather a high incidence of integration in the repeat regions. Similarly, patient B20 (postHCC) had four collections at 3-month intervals, all showing integration in noncoding regions (e.g., simple repeats), although the specific sites varied. Patient B17 (HCC) had two urine collections 2 months apart, with different junction sequences detected. Patient B7, with cirrhosis, had two urine samples collected 7 months apart, with integrations initially detected in a noncoding region followed by integrations in genecoding regions. Interestingly, patient B7 developed HCC 4 years after the second collection. This initial comparison suggests that different $\mathrm{HBV}$ integrations can be captured in urine through serial monitoring. Collectively, our data demonstrate the feasibility of analyzing $\mathrm{HBV}$ integration using urine liquid biopsy. 


\section{TABLE 4. MAJOR HBV INTEGRATIONS IDENTIFIED IN URINE SAMPLES OF PATIENTS INFECTED WITH HBV (STUDY B)}

\begin{tabular}{|c|c|c|c|}
\hline Patient ID & Diagnosis & No. of HBV-JS Species Identified & Major Junctions (\% of Total Junction Reads)* \\
\hline B1 & Hepatitis $(n=5)$ & 48 & Chr4 (5.0\%), SR (4.3\%) \\
\hline $\mathrm{B}^{\dagger}$ & & 0 & $\mathrm{~N} / \mathrm{A}$ \\
\hline B3 & & 2 & Chrl-a (50.0\%), Chr1-b (50.0\%) \\
\hline B4 & & 20 & CHCHD2P9 (20.7\%), HSPE1P8 (17.2\%) \\
\hline B5 & & 10 & SINE (19.0\%), SEMA6D (14.3\%) \\
\hline B6 & Cirrhosis $(n=12)$ & 21 & MLLT4 (10.2\%), FOCAD (8.2\%), PAK3 (8.2\%) \\
\hline$B 7 a^{\ddagger}$ & & 21 & BAGE2 (6.8\%), Chr12 (6.8\%) \\
\hline$B 7 b$ & & 5 & GCSHP2 (20.0\%), PPP2R2C (20.0\%) \\
\hline B8 & & 30 & SR (6.3\%), LINE (4.8\%) \\
\hline B9 & & 10 & SR $(28.0 \%)$ \\
\hline B10 & & 40 & XRCC3 (9.6\%), MST1L (8.0\%) \\
\hline B11 & & 18 & TERT (48.8\%), Chr16 (7.1\%) \\
\hline B12 & & 53 & TERT (30.5\%), GCSHP2 (6.9\%) \\
\hline B13 & & 54 & SR (5.1\%), LINC01481 (4.4\%) \\
\hline B14 & & 13 & AL954742.1 (13.8\%), Chr5 (10.3\%) \\
\hline $\mathrm{B} 15 \mathrm{a}^{\ddagger}$ & & 46 & SINE (13.2\%), LINE (3.9\%) \\
\hline$B 15 b$ & & 58 & SINE (8.0\%), EXD3 (6.8\%) \\
\hline B16 & $\operatorname{HCC}(n=6)$ & 4 & KIF14 (25.0\%), TET (25.0\%) \\
\hline $\mathrm{B} 17 \mathrm{a}^{\ddagger}$ & & 2 & VPS8 (50.0\%); Chr9 (50.0\%) \\
\hline $\mathrm{B} 17 \mathrm{~b}$ & & 5 & SR (20.0\%), AC01 1193.1 (20.0\%) \\
\hline $\mathrm{B} 18$ & & 11 & SR (36.4\%), TERT (9.1\%) \\
\hline $\mathrm{B} 19 \mathrm{a}^{\ddagger}$ & & 17 & CTD-3229J4. 1 (50.8\%), SR (9.2\%) \\
\hline B19b & & 2 & SR-a (50.0\%); SR-b (50.0\%) \\
\hline $\mathrm{B} 20 \mathrm{a}^{\ddagger}$ & Post-HCC $(n=9)$ & 16 & SR (25.0\%), LTR (12.5\%) \\
\hline B20b & & 63 & LMF1-AS1 (5.6\%), hsd17b6 (5.1\%) \\
\hline B2Oc & & 14 & GCSHP2 (60.8\%), SR (10.1\%) \\
\hline B2Od & & 13 & SR (21.4\%), LACTB2-AS1 (7.1\%) \\
\hline B21 & & 4 & SR (75.0\%) \\
\hline B22 & & 13 & TRPM3 (37.0\%), ANKRD9 (13.0\%) \\
\hline B23 & & 2 & PCDHGC5 (50.0\%), HBB (50.0\%) \\
\hline B24 & & 11 & Chr12 (20.7\%), GALNT6 (10.3\%) \\
\hline B25 & & 82 & LINE (12.4\%), LTR (5.8\%) \\
\hline
\end{tabular}

\footnotetext{
* Defined as up to two most abundant junctions with at least two supporting reads.

${ }^{\dagger}$ No one junction sequence meets the major-junction criteria.

${ }^{\ddagger}$ Multiple collections were obtained from the patient.

Abbreviations: AC011193.1, ENSG00000225582.1; AL954742.1, microRNA ENSG00000251912; ANKRD9, ankyrin repeat domain 9; BAGE2, BAGE family member 2; CHCHD2P, coiled-coil-helix-coiled-coil-helix domain containing 2 pseudogene 9; Chr, chromosome location; CTD-3229J4.1, Long Intergenic Non-Protein Coding RNA ENSG00000261555; EXD3, exonuclease 3'-5' domain containing 3; FOCA, focadhesin; GALNT6, polypeptide N-acetylgalactosaminyltransferase 6; GCSHP2, glycine cleavage system protein $\mathrm{H}$ pseudogene 2; HBB, hemoglobin subunit beta; HSD17B6, hydroxysteroid 17-beta dehydrogenase 6; HSPE1P8, heat shock protein family E (Hsp10) member 1 pseudogene 8; KIF14, kinesin family member 14; LACTB2-AS1, LACTB2 antisense RNA 1; LINC0148, long Intergenic Non-Protein Coding RNA 1468; LINE, long interspersed nuclear elements; LMF1-AS1, LMF1 antisense RNA 1; LTR, Long terminal repeat; MLLT4, Myeloid/Lymphoid or Mixed-Lineage Leukemia; MST1L, macrophage stimulating 1 like; N/A, not available; PAK3, p21 (RAC1) activated kinase 3; PCDHGC5, protocadherin gamma subfamily C, 5; PPP2R2C, protein phosphatase 2 regulatory subunit Bgamma; SEMA6D, semaphorin 6; SINE, short interspersed nuclear elements; SR, simple repeat; TERT, telomerase reverse transcriptase; Translocated To, 4; TRPM3, transient receptor potential cation channel subfamily M member 3; VPS8, VPS8 subunit of CORVET complex; XRCC3, X-ray repair cross complementing 3.
} 
A
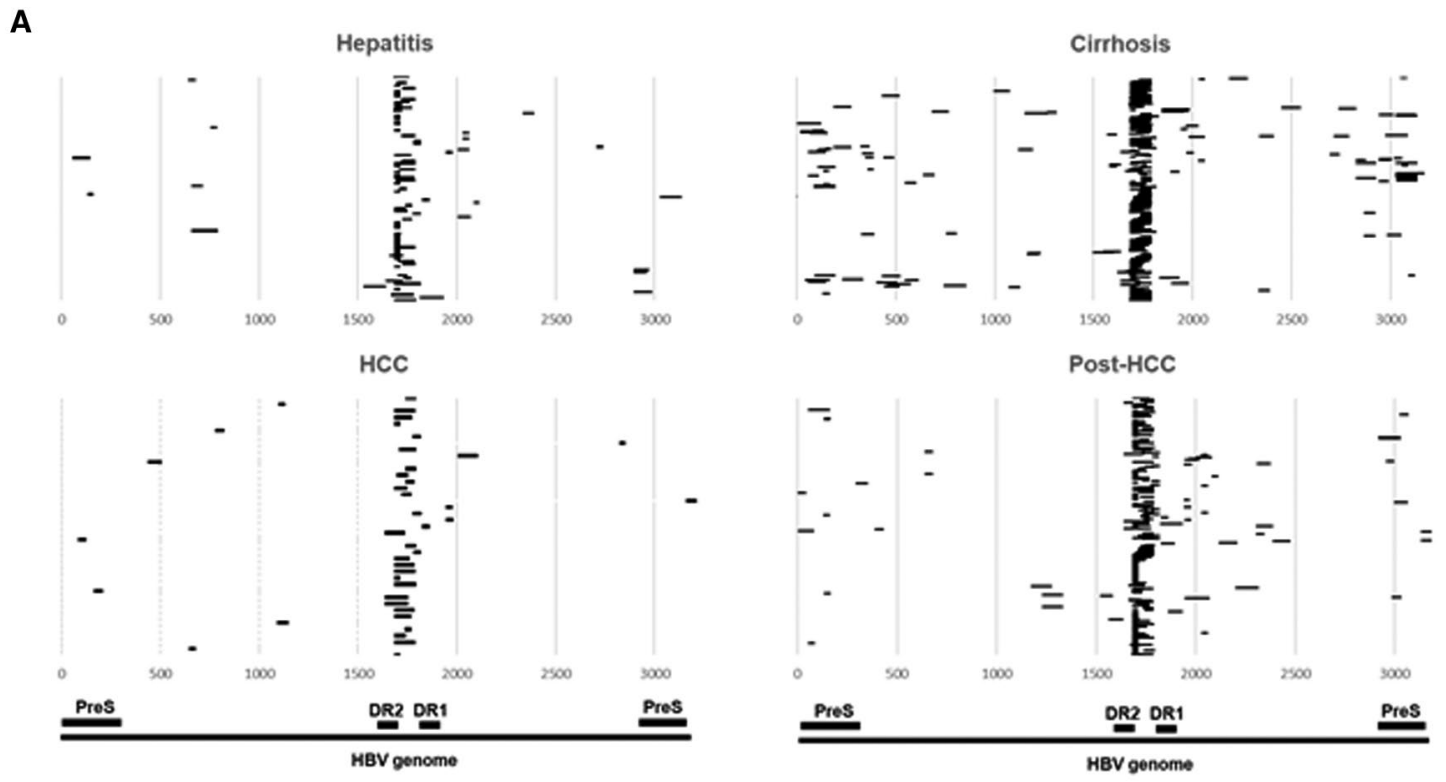

B

\begin{tabular}{|c|c|c|c|c|}
\hline & Hepatitis & Cirrhosis & HCC & Post-HCC \\
\hline Total HBV-JS sequences & 84 & 369 & 41 & 218 \\
\hline Avg S.R per HBV-JS (Range) & $2.74(2-12)$ & $3.06(2-79)$ & $2.76(2-33)$ & $3.97(2-48)$ \\
\hline HBV DR1-2 (\% of total) & $62(73.8 \%)$ & $274(74.2 \%)$ & $29(70.7 \%)$ & $173(79.3 \%)$ \\
\hline PreS1/S2 (\% of total) & $6(7.1 \%)$ & $39(10.5 \%)$ & $2(4.9 \%)$ & $16(7.3 \%)$ \\
\hline Other (\% of total) & $16(19.0 \%)$ & $56(15.7 \%)$ & $10(24.4 \%)$ & $29(13.3 \%)$ \\
\hline
\end{tabular}

FIG. 2. Analysis of HBV integrations in the urine of HBV-infected patients with various liver diseases. (A) Distribution of HBV junction sequence breakpoints across the HBV genome. The HBV sequences indicated by black lines of unique junctions from each patient are plotted by disease category. Totals of 84, 366, 41, and 221 sequences from hepatitis, cirrhosis, HCC, and post-HCC samples, respectively, are shown. (B) Comparison of HBV-DNA breakpoints in HBV DR1-2, PreS1/S2, and other HBV region and the supporting reads per junction detected among the HBV disease categories. Abbreviation: Avg, average.

\section{Discussion}

In this proof-of-concept study, we provide evidence for noninvasive detection of integrated HBV DNA in urine of patients infected with HBV with liver disease ranging from hepatitis to HCC. The importance of this study is threefold. First, we report the detection of integrated HBV DNA in urine. Second, we includes a comparative analysis of $\mathrm{HBV}$ integration in hepatitis, cirrhosis, HCC, and post-HCC using solely ectopic samples. Third, we provide evidence that urine contains liver-derived DNA, by taking advantage of the liver tropism of HBV and unique HBV-JSs as molecular signatures of infected hepatocytes. Taken together, our results demonstrate the potential of urine liquid biopsy for integrated $\mathrm{HBV}$ burden and HBV-related liver disease monitoring.
Our previous study demonstrated that only fragmented HBV DNA was detected in the urine of patients with $\mathrm{HBV}$ infection even when the blood viral load was as high as $10^{8}$ copies/mL. ${ }^{(20)}$ Urine cfDNA is mostly monocleosomal and dinucleosomal in size and largely derived from apoptotic cells. ${ }^{(21)}$ Therefore, an HBV sequence containing urine cfDNA likely originates from degradation of host-integrated HBV genomes protected by nucleosomes in apoptotic $\mathrm{HBV}$-infected hepatocytes. Hepatocytes from the HBV-infected liver have a higher turnover rate than hepatocytes from uninfected normal liver. ${ }^{(38)}$ Thus, urine provides a unique biopsy source to noninvasively detect integrated HBV DNA and monitor infected-cell burden, neither of which is achievable with current clinical HBV tests.

As expected, HBV genome integration breakpoints detected in urine were found to be mostly 
(>70\%) in the HBV DR1-2 region, consistent with previous HBV-HCC tissue studies. ${ }^{(8)}$ Additionally, $11 \mathrm{HBV}-J \mathrm{~S} s$ identified in urine and reported previously in HCC tissue were all found in patients with cirrhosis or HCC. We and others have previously shown that urine contains apoptotic DNA mostly from the circulation, ${ }^{(21)}$ which is expected to include DNA from infected hepatocytes undergoing apoptosis throughout the liver. Therefore, urine DNA is likely to reflect the heterogeneity of HBV-JSs in the liver more accurately compared with a single tumor biopsy or resection. HBV-JSs have been detected in plasma of patients with HCC in recent studies. ${ }^{(12-15)}$ However, because urine cfDNA consists of mostly 1-2-nucleosomal-sized DNA from apoptotic cells, ${ }^{(21)}$ its analysis is less likely to be confounded by the possible presence of any non-nucleosome-associated replicating HBV DNA or virions. ${ }^{(20)}$ As a result, urine is likely to provide a more sensitive liquid biopsy than blood for assessing integrated HBV-DNA sequences in infected patients. Although we did not directly compare matched urine and plasma for HBV-JS detection due to plasma unavailability, the large number of HBV-JSs we detected in urine samples is encouraging. Studies directly comparing urine with matched plasma will be needed to determine which body fluid is better for detection of HBV-JSs in cfDNA.

The detection of HBV-host sequences and a chromosome rearrangement in $\mathrm{HCC}$ urine echoes our recent progress in developing a noninvasive, urinebased HCC screening test. ${ }^{(16,19,39)}$ Characterization of integrated HBV DNA may provide insight into clonal expansion and turnover of infected hepatocytes, HCC risk stratification, monitoring recurrence following treatment, and treatment efficacy. A urine-based test allows routine at-home collection; thus, frequent monitoring can be feasible for patients undergoing antiviral therapy for treatment efficacy evaluation, or for patients at risk of developing primary or recurrent HCC. Characterization of HBV-JSs in urine can be highly applicable in the development of $\mathrm{HBV}$ directed $\mathrm{T}$-cell immunotherapies, as targeting HBV antigens derived from integrated HBV DNA has been shown to have antiviral and antitumor effects. ${ }^{(40,41)}$ Furthermore, detection of urinary integrated HBV sequences may also present a unique opportunity to noninvasively monitor the infected cell burden that may not be captured by current clinical HBV tests. Although more studies are required, the potential of monitoring dynamics of HBV-JS quantity and quality can provide critical molecular profiling to aid in delineating liver pathogenesis in patients with HBV. Because integrations into known HCC drivers were only found in urine of patients with cirrhosis or HCC in this study cohort, characterization of HBV-JSs may hold potential for application in assessing HBVrelated liver disease in infected patients. In the present proof-of-concept study, we demonstrated that detection of HBV-JSs in urine is feasible. Further study with a much larger sample size and quantitative measurement in addition to qualitative characterization is needed to evaluate its potential clinical applications.

\section{REFERENCES}

1) Tu T, Budzinska MA, Vondran FW, Shackel NA, Urban S. Hepatitis B virus DNA integration occurs early in the viral life cycle in an in vitro infection model via NTCP-dependent uptake of enveloped virus particles. J Virol 2018;92:e02007-17.

2) Chauhan R, Churchill ND, Mulrooney-Cousins PM, Michalak TI. Initial sites of hepadnavirus integration into host genome in human hepatocytes and in the woodchuck model of hepatitis B-associated hepatocellular carcinoma. Oncogenesis 2017;6:e317.

3) Chauhan R, Shimizu Y, Watashi K, Wakita T, Fukasawa M, Michalak TI. Retrotransposon elements among initial sites of hepatitis B virus integration into human genome in the HepG2NTCP cell infection model. Cancer Genet 2019;235:39-56.

4) Zhao K, Liu A, Xia Y. Insights into hepatitis B virus DNA integration-55 years after virus discovery. Innovation 2020;1:100034.

5) Shafritz DA, Shouval D, Sherman HI, Hadziyannis SJ, Kew MC. Integration of hepatitis B virus DNA into the genome of liver cells in chronic liver disease and hepatocellular carcinoma. N Engl J Med 1981;305:1067-1073.

6) Matsubara K, Tokino T. Integration of hepatitis $B$ virus DNA and its implications for hepatocarcinogenesis. Mol Biology Med 1990;7:243-260.

7) Bonilla GR, Roberts LR. The role of hepatitis B virus integrations in the pathogenesis of human hepatocellular carcinoma. J Hepatol 2005;42:760.

8) Yan H, Yang Y, Zhang L, Tang G, Wang YuZhao, Xue G, et al. Characterization of the genotype and integration patterns of hepatitis B virus in early-and late-onset hepatocellular carcinoma. Hepatology 2015;61:1821-1831.

9) Li X, Zhang J, Yang Z, Kang J, Jiang S, Zhang T, et al. The function of targeted host genes determines the oncogenicity of HBV integration in hepatocellular carcinoma. J Hepatol 2014;60:975-984.

10) Esumi M, Aritaka T, Arii M, Suzuki K, Tanikawa K, Mizuo H, et al. Clonal origin of human hepatoma determined by integration of hepatitis B virus DNA. Can Res 1986;46:5767-5771.

11) Esumi M, Tanaka Y, Tozuka S, Shikata T. Clonal state of human hepatocellular carcinoma and non-tumorous hepatocytes. Cancer Chemother Pharmacol 1989;23:S1-S3.

12) Li C-L, Ho M-C, Lin Y-Y, Tzeng S-T, Chen Y-J, Pai H-Y, et al. Cell-free virus-host chimera DNA from Hepatitis B virus integration sites as a circulating biomarker of hepatocellular cancer. Hepatology 2020;72:2063-2076.

13) Chen W, Zhang KE, Dong P, Fanning G, Tao C, Zhang H, et al. Noninvasive chimeric DNA profiling identifies tumor-originated 
HBV integrants contributing to viral antigen expression in liver cancer. Hep Intl 2020;14:326-337.

14) Qu C, Wang $Y$, Wang $P$, Chen $K$, Wang $M$, Zeng $H$, et al. Detection of early-stage hepatocellular carcinoma in asymptomatic $\mathrm{HBsAg}$-seropositive individuals by liquid biopsy. Proc Natl Acad Sci 2019;116:6308-6312.

15) Li W, Cui X, Huo Q, Qi Y, Sun Y, Tan M, et al. Profile of HBV integration in the plasma DNA of hepatocellular carcinoma patients. Curr Genomics 2019;20:61-68.

16) Hann H-W, Jain S, Park G, Steffen JD, Song W, Su Y-H. Detection of urine DNA markers for monitoring recurrent hepatocellular carcinoma. Hepatoma Res 2017;3:105-111.

17) Jain S, Xie L, Boldbaatar B, Lin SY, Hamilton JP, Meltzer SJ, et al. Differential methylation of the promoter and first exon of the RASSF1A gene in hepatocarcinogenesis. Hepatology Res 2015;45:1110-1123.

18) Lin SY, Jain S, Song W, Hu C-T, Su Y-H. Strategic assay developments for detection of hbv 1762t/1764a double mutation in urine of patients with $\mathrm{HBV}$-associated hepatocellular carcinomas. In: Hepatocellular Carcinoma-Clinical Research. Chinese University of Hong Kong, China; 2012.

19) Lin SY, Dhillon V, Jain S, Chang T-T, Hu C-T, Lin Y-J, et al. A locked nucleic acid clamp-mediated PCR assay for detection of a p53 codon 249 hotspot mutation in urine. J Mol Diagn 2011;13:474-484.

20) Jain S, Su Y-H, Su Y-P, McCloud S, Xue R, Lee T-J, et al. Characterization of the hepatitis $\mathrm{B}$ virus DNA detected in urine of chronic hepatitis B patients. BMC Gastroenterol 2018;18:40.

21) Su Y-H, Wang M, Brenner DE, Ng A, Melkonyan H, Umansky $\mathrm{S}$, et al. Human urine contains small, 150 to 250 nucleotide-sized, soluble DNA derived from the circulation and may be useful in the detection of colorectal cancer. J Mol Diagn 2004;6:101-107.

22) Su Y-H, Song J, Wang $Z$, Wang $X$, Wang M, Brenner DE, et al. Removal of high molecular weight DNA by carboxylated magnetic beads enhances the detection of mutated K-ras DNA in urine. Ann N Y Acad Sci 2008;1137:82-91.

23) Ding D, Lou X, Hua D, Yu W, Li L, Wang J, et al. Recurrent targeted genes of hepatitis $\mathrm{B}$ virus in the liver cancer genomes identified by a next-generation sequencing-based approach. PLoS Genet 2012;8:e1003065.

24) Shieh F-S, Jongeneel P, Steffen JD, Lin S, Jain S, Song W, et al. ChimericSeq: an open-source, user-friendly interface for analyzing NGS data to identify and characterize viral-host chimeric sequences. PLoS One 2017;12:e0182843.

25) University of Michigan. UM BRCF Bioinformatics Core. https:// github.com/umich-brcf-bioinf/Connor. Accessed June, 2020.

26) Sung W-K, Zheng H, Li S, Chen R, Liu X, Li Y, et al. Genomewide survey of recurrent $\mathrm{HBV}$ integration in hepatocellular carcinoma. Nat Genet 2012;44:765-769.

27) Jiang Z, Jhunjhunwala S, Liu J, Haverty PM, Kennemer MI, Guan Y, et al. The effects of hepatitis B virus integration into the genomes of hepatocellular carcinoma patients. Genome Res 2012;22:593-601.

28) Li W, Zeng XI, Lee NP, Liu X, Chen S, Guo B, et al. HIVID: an efficient method to detect HBV integration using low coverage sequencing. Genomics 2013;102:338-344.

29) Lee WY, Bachtiar M, Choo CC, Lee CG. Comprehensive review of hepatitis B virus-associated hepatocellular carcinoma research through text mining and big data analytics. Biol Rev Camb Philos Soc 2019;94:353-367.

30) Le Pabic H, Bonnier D, Wewer UM, Coutand A, Musso O, Baffet $\mathrm{G}$, et al. ADAM12 in human liver cancers: TGF- $\beta$-regulated expression in stellate cells is associated with matrix remodeling. Hepatology 2003;37:1056-1066.

31) Shuda M, Kondoh N, Imazeki N, Tanaka K, Okada T, Mori $\mathrm{K}$, et al. Activation of the ATF6, XBP1 and grp78 genes in human hepatocellular carcinoma: a possible involvement of the ER stress pathway in hepatocarcinogenesis. J Hepatol 2003;38: 605-614.

32) Zhao J, Ye J, Lin Y, Bu K, Mai R, Liu Z, Gao X, et al. Identification and validation of a ten-gene set variation score as a diagnostic and prognostic stratification tools in hepatocellular carcinoma. Am J Transl Res 2020;12:5683-5695.

33) Zhuang P-Y, Shen J, Zhu X-D, Lu L, Wang L, Tang Z-Y, Sun $\mathrm{H}-\mathrm{C}$. Prognostic roles of cross-talk between peritumoral hepatocytes and stromal cells in hepatocellular carcinoma involving peritumoral VEGF-C, VEGFR-1 and VEGFR-3. PLoS One 2013;8:e64598.

34) Weren RD, Venkatachalam R, Cazier JB, Farin HF, Kets CM, De Voer RM, et al. Germline deletions in the tumour suppressor gene FOCAD are associated with polyposis and colorectal cancer development. J Pathol 2015;236:155-164.

35) Bluemn EG, Spencer ES, Mecham B, Gordon RR, Coleman I, Lewinshtein D, et al. PPP2R2C loss promotes castrationresistance and is associated with increased prostate cancer-specific mortality. Mol Cancer Res 2013;11:568-578.

36) El Kramani N, Elsherbiny NM, El-Gayar AM, Ebrahim MA, Al-Gayyar MMJC. Clinical significance of the TNF- $\alpha$ receptors, TNFRSF2 and TNFRSF9, on cell migration molecules Fascin-1 and Versican in acute leukemia. Cytokine 2018;111:523-529.

37) Wan F, Zhu Y, Han C, Xu Q, Wu J, Dai BO, et al. Identification and validation of an eight-gene expression signature for predicting high Fuhrman grade renal cell carcinoma. Int J Cancer 2017;140: 1199-1208.

38) Summers J, Jilbert AR, Yang W, Aldrich CE, Saputelli J, Litwin S, et al. Hepatocyte turnover during resolution of a transient hepadnaviral infection. Proc Nat Acad Sci 2003;100:11652-11659.

39) Kim AK, Hamilton JP, Lin SY, Chang T-T, Hann H-W, Hu C-T, et al. Urine biomarker: novel approach to hepatocellular carcinoma screening. medRxiv Oncol 2020.

40) Tan AT, Yang N, Lee Krishnamoorthy T, Oei V, Chua A, Zhao $\mathrm{X}$, et al. Use of expression profiles of HBV-DNA integrated into genomes of hepatocellular carcinoma cells to select T cells for immunotherapy. Gastroenterology 2019;156:1862-1876.e1869.

41) Fergusson JR, Wallace Z, Connolly MM, Woon AP, Suckling RJ, Hine DW, Barber C, et al. Immune-mobilizing monoclonal T cell receptors mediate specific and rapid elimination of Hepatitis B-infected cells. Hepatology 2020;72:1528-1540.

\section{Supporting Information}

Additional Supporting Information may be found at onlinelibrary.wiley.com/doi/10.1002/hep4.1783/suppinfo. 